\title{
Uma Revisão da Bibliografia sobre o Ensino da Química Verde
}

\author{
Dominique A. Costa a M. Gabriela T. C. Ribeiro ${ }^{\text {b }}$ e Adélio A. S. C. Machado
}

\begin{abstract}
Apresenta-se uma revisão crítica da bibliografia existente na literatura sobre o ensino da Química Verde, cuja análise e discussão permitiu formular algumas sugestões quanto a pistas que podem levar ao desenvolvimento de actividades pedagógicas úteis para o seu progresso, nomeadamente quanto a experiências laboratoriais.
\end{abstract}

No âmbito da formulação de um plano de doutoramento dirigido à prática experimental da Química Verde (QV) no Ensino Secundário, a ser desenvolvido por um de nós (DAC), procurou aferir-se a extensão actual da penetração daquela nova atitude de realização da química no ensino desta ciência. Neste contexto, realizou-se uma análise sistemática da bibliografia sobre o ensino da QV, com ponto de partida nos artigos publicados no Journal of Chemical Education (JCEd) sobre $\mathrm{o}$ assunto. Esta nota tem por fim apresentar sumariamente os resultados da pesquisa bibliográfica realizada e algumas conclusões genéricas que esta evidenciou, esperando-se que possa ser útil para os colegas que estiverem interessados em fazer evoluir o ensino da química para a QV.

Pesquisa bibliográfica. Para avaliar a importância já atingida pela QV no ensino da química realizou-se uma pesquisa no índice do JCEd [1], feita a partir do termo "green chemistry", sucessivamente nos títulos dos artigos e nas palavras-chave, que identificou 77 artigos (até Dezembro de 2007). Os artigos foram sujeitos a uma análise prévia, verificando-se que 19 não envolvem explicitamente QV (a maioria destes dizem respeito a Química Ambiental). Os 58 restantes [2-59] foram classificados em nove tipos traduzidos pelas seguintes siglas: D, Divulgação da QV; E, Ensino (propostas de ensino, cursos, actividades, etc., sem abordar detalhadamente experiências laboratoriais); ED, Experiência de demonstração; LA, Experiência de laboratório em Química Analítica; LG, Idem

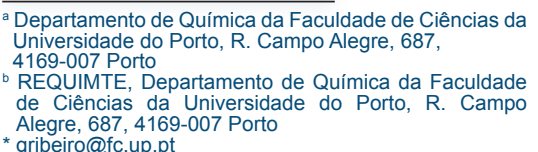
REQUIMTE, Departamento de Química da Faculdade
de Ciências da Universidade do Porto, R. Campo * Alegre, $687,4169-00$ em Química Geral; LI, Idem em Química Inorgânica; LO, Idem em Química Orgânica; M, Métricas; R, Recensão crítica de livro. Esta tarefa conduziu a mais alguns artigos sobre o ensino da QV, na referida revista e noutras [6066], que foram englobados na análise. Por fim, completou-se a pesquisa bibliográfica com a identificação dos artigos de índole pedagógica publicados na revista Green Chemistry da Royal Chemical Society (Londres) [67-74].

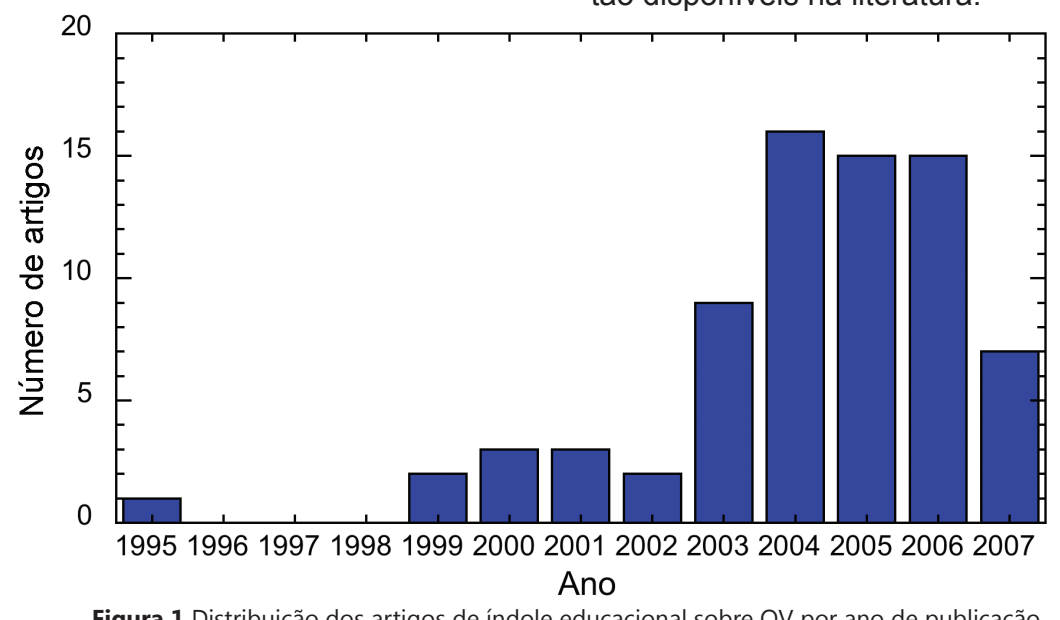

Tabela 1 Distribuição dos artigos* por classes e anos ${ }^{\star \star}$

\begin{tabular}{|c|c|c|c|c|c|c|c|c|c|}
\hline & $\begin{array}{c}\text { D } \\
\text { Divulgação } \\
\text { da QV }\end{array}$ & $\begin{array}{c}\text { E } \\
\text { Ensino } \\
\text { Da QV } \\
\end{array}$ & $\begin{array}{c}\text { ED } \\
\text { Exp. de } \\
\text { demonstração }\end{array}$ & $\begin{array}{c}\text { LA } \\
\text { Exp. Lab } \\
\text { Q. Anal. }\end{array}$ & $\begin{array}{c}\text { LG } \\
\text { Exp. Lab. } \\
\text { Q. Geral } \\
\end{array}$ & $\begin{array}{c}\text { LI } \\
\text { Exp. Lab } \\
\text { Q. Inorg } \\
\end{array}$ & $\begin{array}{c}\text { LO } \\
\text { Exp. Lab. } \\
\text { Q.Org } \\
\end{array}$ & $\begin{array}{c}\text { M } \\
\text { Métricas }\end{array}$ & \begin{tabular}{|c|}
$\mathbf{R}$ \\
$\begin{array}{c}\text { Recensão } \\
\text { de livro }\end{array}$ \\
\end{tabular} \\
\hline 1995 & 2 & & & & & & & & \\
\hline 1999 & 3 & & & & & 4 & 4 & & \\
\hline 2000 & 5 & & & & & 60 & 6 & & \\
\hline 2001 & 7 & & & & & & 61,67 & & \\
\hline 2002 & & & & & & & 8,9 & & \\
\hline 2003 & & 68 & 12 & 10 & 69 & & $\begin{array}{c}62,63,64 \\
68\end{array}$ & 62 & 11,13 \\
\hline 2004 & 14,70 & $\begin{array}{l}18,20, \\
21,65\end{array}$ & 71 & & & 15,16 & $\begin{array}{l}17,19,21 \\
22,23,24 \\
65,66,72\end{array}$ & & \\
\hline 2005 & & $\begin{array}{r}30,35, \\
73,74\end{array}$ & & & 25 & 32 & $\begin{array}{l}26,27,28 \\
29,31,33 \\
34,36,37\end{array}$ & $27,35,73$ & \\
\hline 2006 & 47 & 45,48 & & & 45 & & $\begin{array}{c}39,40,41 \\
42,43,44 \\
46,49,50 \\
51,52\end{array}$ & 45 & 38 \\
\hline 2007 & 54,55 & 55 & & & & & $\begin{array}{c}53,56,57 \\
58\end{array}$ & 59 & \\
\hline Total & 9 & 12 & 2 & 1 & 3 & 5 & 43 & 6 & 3 \\
\hline
\end{tabular}

*) Referências: em tipo normal, do JCEd; em itálico, da revista Green Chemistry; em negrito, de outras revistas.

${ }^{\star}$ ) Referencias: em tipa nomes completos da siglas, ver o texto.

Estes dois grupos de artigos foram classificados como os anteriores. Nas Tabela 1 e Figura 1 apresentam-se, respectivamente, os resultados da classificação (número total de artigos em cada uma das nove classes), e o número total de artigos publicados em cada ano. A Tabela 1 e as referências bibliográficas permitirão aos interessados no ensino da QV orientar-se quanto à escolha de informação, em especial, trabalhos práticos, que já estão disponíveis na literatura. 
Emergência e alcance da QV. A distribuição anual dos artigos na Figura 1 mostra que o primeiro artigo sobre 0 ensino da QV foi publicado no JCEd em 1995 [2]. Tratava-se de um artigo de divulgação, que apresenta um curso introdutório à QV; segundo o que o conteúdo programático e a bibliografia de suporte sugerem, só 9 lições (sobre catálise e biocatálise) das 25 do curso seriam dirigidas à QV - as outras contemplavam tópicos clássicos de química ambiental, poluição e sua remediação, embora procurando seguir uma estratégia global de QV (o artigo foi publicado antes da divulgação dos Doze Princípios da QV [75]). Os autores mencionam designações alternativas para $\mathrm{QV}$, por exemplo "química ambientalmente benigna", mas afirmam que QV é "a mais largamente usada" - esta afirmação refere-se possivelmente a uso coloquial, já que na literatura científica e educacional o termo era então ainda raramente empregue [76].

Só quatro anos depois, em 1999, surgem no JCEd mais dois artigos sobre QV. Um deles [3] refere o uso dos conteúdos das propostas premiadas anualmente no concurso anual da Presidential Green Chemistry Challenge [77], lançada pelo presidente Clinton em 1995, para uma actividade docente destinada a propagandear a QV em cursos de Química Ambiental e Química Industrial. ${ }^{1} \mathrm{O}$ outro artigo [4] analisa a complementaridade da Química à Microescala relativamente à $Q V$ e mostra de modo qualitativo, sem quantificar com métricas, como alguns aspectos da prática da primeira cumprem objectivos da segunda, por exemplo: produção de menos resíduos (e menores gastos de reagentes), uso de reagentes e/ou solventes alternativos menos perigosos, utilização de novas vias de síntese, menores tempos de reacção, etc.

Nos anos seguintes, até 2002, foram publicados na literatura 2-4 artigos por ano, verificando-se depois uma subida rápida para 15-16 artigos por ano a partir de 2004. No entanto, interessantemente, em 2007 o número decresceu para 7. Este facto pode ser casual ou significar um certo "grau de descrença" na QV resultante da aplicação imprópria do termo, já que não é infrequente a "reivindicação de verdura" ser ilegítima: por vezes, os químicos melhoram a verdura de uma reacção numa área, mas esquecem as outras, piorando a verdura global (a ref. [78] apresenta um exemplo recente que elucida esta afirmação).

Quanto ao alcance da bibliografia da QV, avaliado pela temática dos 73 artigos analisados, a Tabela 1 mostra que cerca de um terço se refere à divulgação e ensino (24 artigos nas classes $D, E$ e R), apenas 6 contemplam a apresentação e cálculo de métricas (classe M), e mais de dois terços (54 artigos) propõem a realização de demonstrações e experiências de QV (classes ED, LA, LG, LI e LO). Quanto a estes últimos, a grande maioria (43 artigos) refere-se a trabalhos práticos de Química Orgânica - evidenciando o menor interesse que tem havido na preparação de experiências de demonstração (2 artigos) e de laboratórios de Química Geral (3 artigos) e Química Inorgânica (5 artigos) dirigidas à $Q V$.

Discussão. A análise realizada permitiu definir algumas linhas de actuação que devem ser seguidas para se desenvolver o ensino da QV, nomeadamente por via experimental.

Um primeiro facto a realçar é o maior esforço que deve ser feito quanto à preparação de experiências de demonstração e de trabalhos práticos adequados para laboratórios de nível introdutório - são necessárias mais experiências que possibilitem primeiros contactos com a QV, para a instilar mais largamente, e de raiz, na mente dos jovens estudantes.

Outro aspecto que merece menção é a total ausência de experiências dirigidas à Química Industrial. A química é o único ramo da ciência que tem uma indústria específica associada a ele a Indústria Química, cuja actividade é fulcral quando se consideram os seus efeitos no ambiente e a necessidade de incrementar a verdura da química - por isso, até para estimular o interesse pelas actividades industriais da química, devem implementar-se experiências que mostrem as possibilidades de esverdeamento dos processos industriais. A maioria dos artigos sobre experiências de laboratório tem vindo a ser dirigida à Química Orgânica - o que não é de espantar, porque como mostram os valores do Factor E [79] é neste ramo da química que o problema da baixa produtividade atómica [80] mais intensamente se põe. De qualquer modo, esta situação põe aos químicos inorgânicos o desafio de proporcionarem mais experiências de QV.

Por outro lado, com excepção de seis artigos $[27,35,45,59,62,73]$ que incluem o cálculo de métricas de massa para aferir a qualidade de verdura química alcançada nas experiências propostas ou em reacções, esta é avaliada, frequentemente, de forma algo vaga e subjectiva, pelo cumprimento de alguns dos Doze Princípios da QV - e mesmo nos artigos em que há o cuidado de explicar como foi conseguido um ganho de verdura, a justificação é vulgarmente feita por afirmações qualitativas simplistas como as exemplificadas no Quadro 1 (por ex. [4,10,15,24,41,52,55,58]). Os Doze Princípios são dirigidos aos químicos académicos de laboratório e têm-se mostrado muito úteis para propagandear a QV e ajudar a perseguir os objectivos desta, mas a química é uma actividade muito diversificada e complexa e, por isso, a variedade de situações encontradas no mundo real a requererem modificações para aumentar a sua benignidade não é "governada" apenas por eles. Em particular, os Doze Princípios não contemplam especificamente, e não permitem aferir, a prática industrial da química, que desde sempre, por razões de segurança, procurou processos o mais benignos possível (Alfredo Nobel foi talvez o primeiro químico verde! [81]); nomeadamente, o desenvolvimento dos processos químicos exige um conjunto de ferramentas variadas que permitam avaliações comparativas de alternativas de processos e operações, por métricas quantitativas diversas, referentes às diferentes características com relevância ambiental das substâncias e reacções químicas [82]. Esta situação sugere que se deve dar mais atenção à incorporação de métricas quantitativas de verdura nas experiências laboratoriais de $\mathrm{QV}$, embora isso nem sempre seja fácil. A propósito da importância das métricas, aconselha- 
-se a leitura de um artigo recente que mostra os progressos que o cálculo do Factor E trouxe para esverdear a Química Industrial [83].

\section{Quadro 1}

Justificações mais vulgares da verdura alcançada nas novas experiências propostas

Redução de...

Quantidades e custos de reagentes estequiométricos e auxiliares

(reagentes protectores, solventes, agentes de separação, etc.)

Quantidades de resíduos produzidos e correspondentes custos de deposição Tempos de reacção

Toxicidade (reagentes, solventes, etc.)

Energia dispendida (reacções à temperatura ambiente)

Solventes orgânicos (por realização da reacção em meio aquoso ou sem solvente)

Aumento de...

Segurança (eliminação de solventes tóxicos e inflamáveis e preferência pela água) Facilidade do isolamento e acabamento do produto ("work-up") Economia atómica

\section{Outros}

Catalisador de origem renovável e biodegradável Prevenção de resíduos em vez de tratamento

Embora muitos dos artigos realcem a postura de criatividade e inovação que as experiências de QV permitem instilar na mente dos alunos, o que seguramente é de enaltecer, as experiências são invariavelmente apresentadas com atitude académica, tradicionalmente reducionista. A ideia básica da QV é tão apelativa do ponto de vista da Sustentabilidade, que "foi agarrada" com vigor pela comunidade química académica com consciência ambiental, mas a sua prática exige uma mudança de atitude por parte dos químicos, que acompanhe a da sociedade quanto ao Desenvolvimento Sustentável - requer a adopção de um paradigma de pensamento holístico, que considere como unidade básica de raciocínio o ciclo-de-vida do composto e do processo usado para o seu fabrico, considerando o seu alcance global, mesmo quando se considera apenas um detalhe do ciclo (só com esta nova atitude se impede o aparecimento das falsas "reivindicações de verdura" referidas atrás).

\section{Conclusão}

A discussão anterior sugere que é preciso fazer sentir aos estudantes a necessidade de, tanto quanto possível, aferir quantitativamente os ganhos (ou perdas) de verdura global, em todo o plo, uma reacção química intencionalmente verde pode facilmente "ser estragada" por um acabamento de produto ("work-up") sem verdura. Em face da já referida complexidade da química, sente-se que há lugar para a procura de novos trabalhos práticos para o ensino da QV, não só para o ensino universitário, mas também para o ensino secundário, que contemplem conjuntamente a implementação de novos procedimentos laboratoriais verdes e a avaliação dos seus ganhos de verdura por cálculo de métricas, quer estequiométricas (produtividade do uso dos átomos) [80], quer ambientais, mediante a inclusão de ferramentas de aferição relativa adequada - nomeadamente, um desafio concreto a atacar será incluir métricas adequadas de verdura ambiental nas actividades de laboratório.

\section{Nota}

${ }^{1}$ De referir que as propostas concorrentes à Presidential Green Chemistry Challenge devem contemplar situações com verdura comprovada por demonstração cabal ou prática no mundo real (nos EUA, nos cinco ano anteriores ao de atribuição).

\section{REFERÊNCIAS}

[1] J. Chem. Ed., em http://jchemed.med.
wisc.edu/Journal/Search/search.htm (acedido em 2007.11.26)

[2] T.C. Collins, "Introduction Green Chemistry in Teaching and Research", J. Chem. Ed. 72 (1995) 965-6.

[3] M. C. Cann, "Bringing State-of-the Art, Applied, Novel, Green Chemistry to the Classroom by Employing the Presidential Green Chemistry Challenge Awards", J. Chem. Ed. 76 (1999) 163941.

[4] M. M. Singh, Z. Szafran e R.M. Pike, "Microscale Chemistry and Green Chemistry: Complementary Pedagogies", J. Chem. Ed. 76 (1999) 1684-6.

[5] D. L. Hjeresen, D. L. Schutt e J. M. Boese, "Green Chemistry and Education”, J. Chem. Ed. 77 (2000) 1543-7.

[6] S. M. Reed e J. E. Hutchison, "Green Chemistry in the Organic Teaching Laboratory: An Environmental Benign Synthesis of Adipic Acid", J. Chem. Ed. 77 (2000) 1627-9.

[7] M. M. Kirchhoff, "Topics in Green Chemistry”, J. Chem. Ed. 78 (2001) 1577.

[8] N. Pohl, A. Clague e K. Schwarz, "Chiral Componds and Green Chemistry in Undergraduate Organic Laboratories: Reduction of a Ketone by Sodium Borohydride and Baker's Yeast", J. Chem. Ed. 79 (2002) 727-8

[9] B. A. Harper, J. C. Rainwater, K. Birdwhistell e D. A. Knight, "Aqueous-Phase Palladium-Catalysed Coupling - A Green Chemistry Laboratory Experiment", J. Chem. Ed. 79 (2002) 729-31.

[10] D. L. Giokas, E. K. Paleologos e M. I. Karayannis, "Micele-Mediated Extraction of Heavy Metals from Environmental Samples - An Environmental Green Chemistry Laboratory Experiment", J. Chem. Ed. 80 (2003) 61-4.

[11] W. Canover, "Introduction to Green Chemistry" (M. A. Ryan e M. Tinnesand (eds.), ACS, 2002), J. Chem. Ed. 80 (2003) 268.

[12] W. E. Wellman e M. E. Noble, "Greening the Blue Bottle", J. Chem. Ed. 80 (2003) 537-40.

[13] A. M. Rosan, "Green Chemistry: An Introductory Text" (M. Lancaster, RSC, 2002), J. Chem. Ed. 80 (2003) 1141-2.

[14] E. S. Uffelman, "News from Online: Green Chemistry", J. Chem. Ed. 81 (2004) 172-6.

[15] E. S. Uffelman, J. R. Doherty, C Schulze, A. L. Burke, K. R. Bonnema, T. T. Watson e D. W. Lee III, "Microscale Syntheses, Reactions, and $1 \mathrm{H}$ NMR Spectroscopic Investigations of Square Planar Macrocyclic Tetraamido-N Cu(III) 
Complexes Relevant to Green Chemistry", J. Chem. Ed. 81 (2004) 182-5.

[16] E. S. Uffelman, J. R. Doherty, C. Schulze, A. L. Burke, K. R. Bonnema, T. T. Watson e D. W. Lee III, "Microscale Syntheses, Reactions, and $1 \mathrm{H}$ NMR Spectroscopic Investigations of Square Planar Macrocyclic Tetraamido-N Co(III) Complexes Relevant to Green Chemistry", J. Chem. Ed. 81 (2004) 325-9.

[17] A. J. Seen, "Alkene Isomerization Using a Solid Acid as Activator and Support for a Homogenous Catalyst", J. Chem. Ed. 81 (2004) 383-4.

[18] Y. Song, Y. Wang e Z. Geng, "Some Exercices Reflecting Green Chemistry Concepts", J. Chem. Ed. 81 (2004) 691-2.

[19] F. Fringuelli, O. Piermatti e F. Pizzo, "One-Pot Synthesis of 7-Hydroxy-3carboxycoumarin in Water", J. Chem. Ed. 81 (2004) 874-6.

[20] M. C. Cann e T. A. Dickneider, "Infusing the Chemistry Curriculum with Green Chemistry Using Real-World Examples, Web Modules, and Atom Economy in Organic Chemistry Courses", J. Chem. Ed. 81 (2004) 977-80.

[21] T. E. Goodwin, "An Asymptotic Approach to the Development of a Green Organic Chemistry Laboratory", J. Chem. Ed. 81 (2004) 1187-90.

[22] D. R. Palleros, "Solvent-Free Synthesis of Chalcones", J. Chem. Ed. 81 (2004) 1345-7.

[23] S. H. Leung e S. A. Angel, "SolventFree Wittig Reaction: A Green Organic Chemistry Laboratory Experiment", J. Chem. Ed. 81 (2004) 1492-3.

[24] J. J. Esteb, K. M. Gligorich, S. A. O'Reilly e J. M. Richter, "Solvent Free Conversion of a-Naphtaldehyde to 1-Naphthoic Acid and 1-Naphthalenemethanol: Application of the Cannizzaro Reaction", J. Chem. Ed. 81 (2004) 1794-5.

[25] S. M. McCarthy e S. W. Gordon-Wylie, "A Greener Approach for Measuring Colligative Properties", J. Chem. Ed. 82 (2005) 116-9.

[26] J. M. Daley e R. G. Landolt, "A Substitute for "Bromine in Carbon Tetrachloride", J. Chem. Ed. 82 (2005) 120-1.

[27] L. C. McKenzie, L. M. Huffman e J. E. Hutchison, "The Evolution of a Green Chemistry Laboratory Experiment: Greener Brominations of Stilbene", J. Chem. Ed. 82 (2005) 306-10.

[28] G. W. V. Cave e C. L. Raston, "Green Chemistry Laboratory: Benign Synthesis of 4,6-Diphenyl(2,2')bipyridine via Sequential Solventless Aldol and Michael Addition Reactions", J. Chem. Ed. 82 (2005) 468-9.

[29] T M. Jones-Wilson e E. A. Burtch, "A Green Starting Material for Electrophilic Aromatic Substitution for the Undergraduate Organic Laboratory", J. Chem. Ed. 82 (2005) 616-7.

[30] J. A. Haack, J. E. Hutchison, M. M. Kirchhoff e I. J. Levy, "Going Green: Lecture Assignments and Lab Experiences for the College Curriculum", J. Chem. Ed. 82 (2005) 974-6.

[31] L. L. White e K. W. Kittredge, "A Microwave Assisted Reduction of Cyclohexanone Using Solid-State Supported Sodium Borohydride", J. Chem. Ed. 82 (2005) 1055-6.

[32] A. Romero, G. Hernández e M. F. Suárez, "Photocatalytic Oxidation of Sulfurous Acid in na Aquesous Medium", J. Chem. Ed. 82 (2005) 1234-6.

[33] G. D. Bennett, "A Green Polymerization of Aspartic Acid for the Undergraduate Organic Laboratory", J. Chem. Ed. 82 (2005) 1380-1.

[34] T. Friscic, T. D. Hamilton, G. P. Papaefstathiou e L. R. MacGillivray, "A Template-Controlled Solid-State Reaction for the Organic Chemistry Laboratory", J. Chem. Ed. 82 (2005) 1679-81.

[35] S. D. Van Arnum, "An Approach Towards Teaching Green Chemistry Fundamentals", J. Chem. Ed. 82 (2005) 1689-92.

[36] J. J. Esteb, J. N. Hohman, D. E. Schlamadinger e A. M. Wilson, "A Solvent Free Baeyer-Villiger Lactonization for the Undergaduate Organic Laboratory: Synthesis of $\mathrm{y}$-t-Butyl- $\varepsilon$-caprolactone", J. Chem. Ed. 82 (2005) 1837-8.

[37] G. A. Sereda, "Comparative Methylation of 1,8-Dihydroxy-9,10-antraquinone: Chemoselectivity in the Organic Chemistry Laboratory", J. Chem. Ed. 82 (2005) 1839-40.

[38] R. Pagni, "Environmental Chemistry", $3^{a}$ ed (C. Baird e M. Cann, Freeman, 2005), J. Chem. Ed. 83 (2006) 217.

[39] R. L. Crumbie, "Environmentally Responsible Redox Chemistry: An Example of Convenient Oxidation Methodology without Chromium Waste", J. Chem. Ed. 83 (2006) 268-9.

[40] M. R. Dintzner, P. R. Wucka e T. W. Lyons, "Microwave-Assisted Synthesis of a Natural Insecticide on Basic Montmorillonite K10 Clay", J. Chem. Ed. 83 (2006) 270-2.

[41] I. Montes, D. Sanabria, M. Garcia, J. Castro e J. Fajardo, "A Greener Ap- proach to Aspirin Synthesis Using Microwave Irradiation", J. Chem. Ed. 83 (2006) 628-31.

[42] R. Musiol, B. Tyman-Szram e J. Polanski, "Microwave-Assisted Heterocyclic Chemistry for the Undergraduate Organic Laboratory", J. Chem. Ed. 83 (2006) 632-3.

[43] K. M. Touchette, "Reductive Amination: A Remarkable Experiment for the Organic Laboratory", J. Chem. Ed. 83 (2006) 929-30.

[44] K. K. W. Mak, J. Siu, Y. M. Lai e P. Chan, "Mannich reactions in Room Temperature Ionic Liquids (RTILs): An Advanced Undergarduate Project of Green Chemistry and Structural Elucidation", J. Chem. Ed. 83 (2006) 943-6.

[45] K. L. Cacciatore e H. Sevian, "Teaching Lab Report Writing through Inquiry: A Green Chemistry Stoichiometry Experiment for General Chemistry", J. Chem. Ed. 83 (2006) 1039-41.

[46] S. Ravia, D. Gamenara, V. Scjhapiro, A. Bellomo, J. Adum, G. Seoane e D. Gonzalez, "Enantioselective Reduction by Crude Plant Parts: Reduction of Benzofuran-2-yl Methyl Ketone with Carrot (Daucus carota) Bits", J. Chem. Ed. 83 (2006) 1049-51.

[47] J. W. Moore, "Faculty Responsabilities", J. Chem. Ed. 83 (2006) 1111.

[48] B. Braun, R. Charney, A. Clarens, J. Farrugia, C. Kitchens, C. Lisowski, D. Nainstat e A. O'Neil, "Completing Our Education: Green Chemistry in the Curriculum", J. Chem. Ed. 83 (2006) 1126-9.

[49] J. Bennett, K. Meldi e C. Kimmell II, J. Chem. Ed. 83 (2006) 1221-4.

[50] J. Pereira e C. A. M. Afonso, "Asymetric Aldol Reaction Induced by Chiral Auxiliary", J. Chem. Ed. 83 (2006) 1333-5.

[51] A. J. F. N. Sobral, "Synthesis of mesoDiethyl-2,2'-dipyrromethane in Water", J. Chem. Ed. 83 (2006) 1665-6.

[52] G. D. Bennett, "A Green Enantioselective Aldol Condensation for the Undergraduate Organic Laboratory", J. Chem. Ed. 83 (2006) 1871-2.

[53] K. C. Nguyen e H. Weizman, "Greening Witting Reactions: Solvent-Free Synthesis of Ethyl trans-Cinnamate and trans-3-(9-Antrhyl)-2-Propenoic Acid Ethyl Ester", J. Chem. Ed. 84 (2007) 119-21.

[54] E. S. Uffelman, "News from Online: Renewable Resources", J. Chem. Ed. 84 (2007) 220-2.

[55] A. E. Marteel-Parrish, "Toward the Greening of Our Minds: A New Special 
Topics Course", J. Chem. Ed. 84 (2007) 245-7.

[56] R. D. Crouch, A. Richardson, J. L. Howard, R. L. Harker e K. H. Barker, "The Aldol Addition and Condensation: The Effect of Conditions on Reaction Pathway", J. Chem. Ed. 84 (2007) 475-6.

[57] G. A. Sereda e V. B. Rajpara, "A Green Alternative to Aluminum Chloride Alkylation of Xylene", J. Chem. Ed. 84 (2007) 692-3.

[58] R. Gandhari, P. P. Maddukuri e T. K. Vinod, "Oxidation of Aromatic Aldehydes Using Oxone", J. Chem. Ed. 84 (2007) 852-4.

[59] J. Andraos e M. Sayed, "On the Use of "Green" Metrics in the Undergraduate Organic Chemistry Lecture and Lab to Assess the Mass Efficiency of Organic Reactions", J. Chem. Ed. 84 (2007) 1004-10.

[60] Z. Zafran, M. M. Singh e R. M. Pike, "The Philosophy of Green Chemistry as Applied to the Microscale Inorganic Chemistry Laboratory", Educación Química 11 (2000) 172-3.

[61] M.Hulce e D. W. Maks, "Organic-Solvent-Free Phase-Transfer Oxidation of Alcohols Using Hydrogen Peroxide", J. Chem. Ed. 78 (2001) 66-7.

[62] L. M. O. C. Merat e R. A. S. San Gil, "Inserção do Conceito de Economia Atómica no Programa de uma Disciplina de Química Orgânica Experimental", Química Nova 26 (2003) 779-81.

[63] J. J. Esteb e M. B. Stockton, "A Solvent-Free Claisen Condensation Reaction for the Organic Laboratory", J. Chem. Ed. 80 (2003) 1446-7.

[64] J. J. Esteb, M. W. Schelle e A. M.
Wilson, "A Solvent-Free Oxidation of Alcohols in an Organic Laboratory", J. Chem. Ed. 80 (2003) 907-8.

[65] E. S. Santos, I. C. G. Garcia e E. F. L. Gomez, "Caring for the Environment While Teaching Organic Chemistry”, J. Chem. Ed. 81 (2004) 232-8.

[66] L. C. McKenzie, L. M. Huffman, K. E. Parent, J. E. Hutchison e J. E. Thompson, "Patterning Self-Assembled Monolayers on Gold", J. Chem. Ed. 81 (2004) 545-8.

[67] M. G. Warner, G. L. Succaw e J. E. Hutchison, "Solventless Syntheses of Mesotetraphenylporphyrin: New Experiments for a Greener Organic Chemistry Laboratory Curriculum", Green Chem. 3 (2001) 267-70

[68] S. Tavener, J. Hardy, N. Hart e A. Goddard, "Teaching Green Chemistry: From Lemons to Lemonade Bottles", Green Chem. 5 (2003) G46-8.

[69] A. F. Houri e H. Wehbe, "Towards an environmentally Friendly Chemistry Laboratory: Managing Expired Chemicals", Green Chem. 5 (2003) G49-50.

[70] W. Leitner, "Focus on Education in Green Chemistry", Green Chem. 6 (2004) 351.

[71] P. Licence, D. Litchfield, M. P. Dellar e M. Poliakoff, " "Supercriticality": A Dramatic But Safe Demonstration of the Critical Point", Green Chem. 6 (2004) 352-4.

[72] L. C. McKenzie, J. E. Thompson, R.Sullivan e J. E. Hutchison, "Green Chemical Processing in the Teaching Laboratory: A Convenient Liquid $\mathrm{CO}_{2}$ Extraction of Natural Products", Green Chem. 6 (2004) 355-8.

[73] S. Grant, A. A. Freer, J. M. Winfield
C. Gray e D. Lennon, "Introducting Undergraduates to Green Chemistry: An Interactive Teaching Exercise", Green Chem. 7 (2005) 121-8.

[74] S. L. Y. Tang, R. L. Smith e M. Poliakoff, "Principles of Green Chemistry: PRODUCTIVELY", Green Chem. 7 (2005) 761-2.

[75] P. T. Anatas e J. C. Warner, Green Chemistry - Theory and Practice, Oxford UP (1998)

[76] E. J. Woodhouse e S. Breyman, "Green Chemistry as Social Movement?", Sci. Techn. \& Hum. Values $\mathbf{3 0}$ (2005) 199-22.

[77] "Presidential Green Chemistry Challenge", em http://www.epa.gov/gcc/ pubs/pgcc/presgcc.html (acedido 2007.12.07)

[78] D. G. Blackmond, A. Armstrong, V. Coombe e A. Wells, "Water in Organocatalytic Processes: Debunking the Myths", Angew. Chem. Int. Ed. 46 (2007) 3798-800.

[79] R. A. Sheldon, "Organic Synthesis Past, Present and Future", Chem. Ind. (London) 23 (1992) 903-6.

[80] A. A. S. C. Machado, "Métricas da Química Verde - A Produtividade Atómica", Boletim SPQ, 107 (2007) 47-55.

[81] A. A. S. C. Machado, "Alfred Nobel O "Primeiro Químico Verde"?", Boletim $S P Q, 103$ (2006) 45-55.

[82] D. T. Allen e D. R.Shonnard, Green Engineering - Environmentally Conscious Design of Chemical Processes, Prentice-Hall (2002)

[83] R. A. Sheldon, "The E Factor: Fifteen Years On”, Green Chem. 9 (2007) 1273-83.

\section{Actualidades Científicas}

A Konarka anunciou em Março último a primeira produção de células solares orgânicas por impressão com jacto de tinta. $\mathrm{O}$ anúncio foi feito nas páginas da revista Advanced Materials no artigo " High Photovoltaic Performance of Inkjet Printed Polymer: Fullerene Blends» e demonstra que é possível fabricar células fotovoltaicas de plástico em larga escala e em ambiente pouco exigente.

A deposição dos polímeros utilizados pode ser feita em substratos de grandes dimensões e em produção contí- nua na forma de rolos, sem exigência dos caros ambientes limpos necessários à maioria das tecnologias que envolvem semicondutores.

Embora as células fotovoltaicas orgânicas apresentem actualmente menores eficiências que as células solares de silício, encontram mercado em nichos de baixa exigência de energia.

O seu baixo custo de produção, especialmente quando comparado com os análogos das células fotovoltaicas inorgânicas tradicionais, tornam-nas bastante atractivas para utilizações como a alimentação de sensores ou de lâmpadas auxiliares.

http://www.konarka.com/index.php/site/ press/konarka_announces_first_ever_ demonstration_of_inkjet_printed_solar_ cells 
Most accessed articles

9/2006-8/2007

Cu'-Catalyzed Alkyne-Azide "Click"

Cycloadditions from a Mechanistic and

Synthetic Perspective

V. D. Bock, H. Hiemstra,

J. H. van Maarseveen

Eur. J. Org. Chem. 2006, pp. 51-68

Recent Advances in Asymmetric

Organocatalytic 1,4-Conjugate Additions

S. B. Tsogoeva

Eur. J. Org. Chem. 2007, pp. 1701-1716

Asymmetric Ring-Opening of Epoxides and Aziridines with Carbon Nucleophiles M. Pineschi

Eur. J. Org. Chem. 2006, pp. 4979-4988

Gold-Catalyzed Hydroamination of $\mathrm{C}-\mathrm{C}$ Multiple Bonds

R. A. Widenhoefer, X. Han

Eur. J. Org. Chem. 2006, pp. 4555-4563

Organocatalytic Synthesis of Drugs and

Bioactive Natural Products

R. M. de Figueiredo, M. Christmann

Eur. J. Org. Chem. 2007, pp. 2575-2600

Articles were downloaded ca. 500,000

times in the last 12 months by scientists in over 120 countries. EurJOC is among the top ten most frequently visited journals in Wiley InterScience.

For more information please visit:

www.eurjoc.org

Subscribe now!

Please send an e-mail to:

cs-journals@wiley.com

(North and South America)

service@wiley-vch.de

(Germany/Austria/Switzerland)

cs-journals@wiley.co.uk

(all other areas)

\section{WILEY}

InterScience ${ }^{\circ}$

(2)WILEY-VCH

\section{Made in Europe Eurroc} for the World

\section{Geographical distribution of published articles 2006}
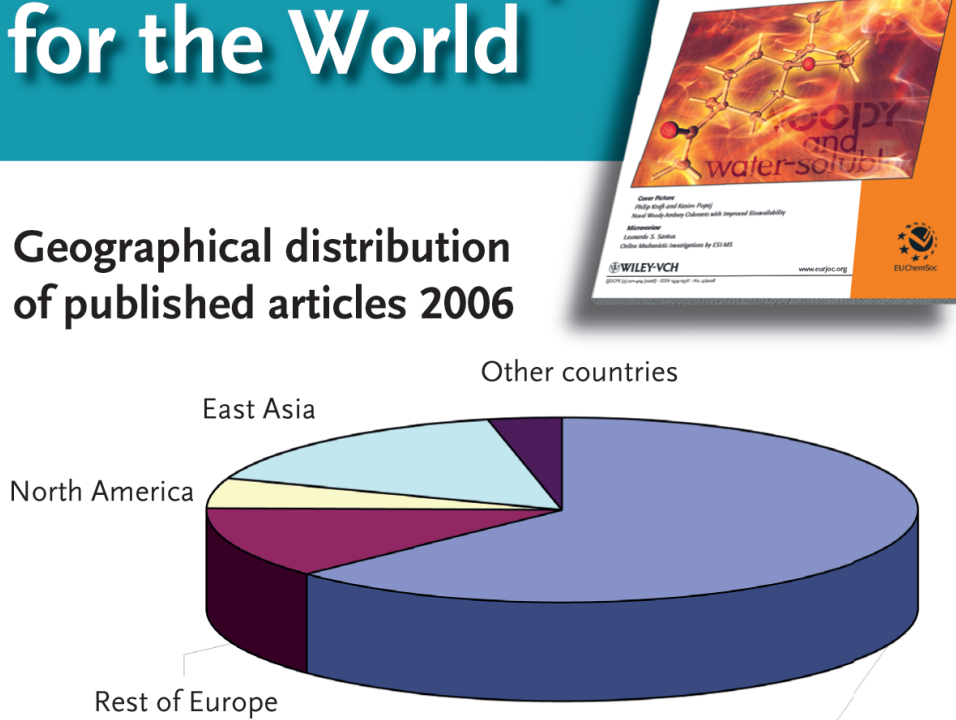

EUChemSoc partner countries

\section{Manuscripts received 2001-2006}

Since 2002, EurJOC has seen an average yearly increase of $11 \%$ in manuscripts submitted.

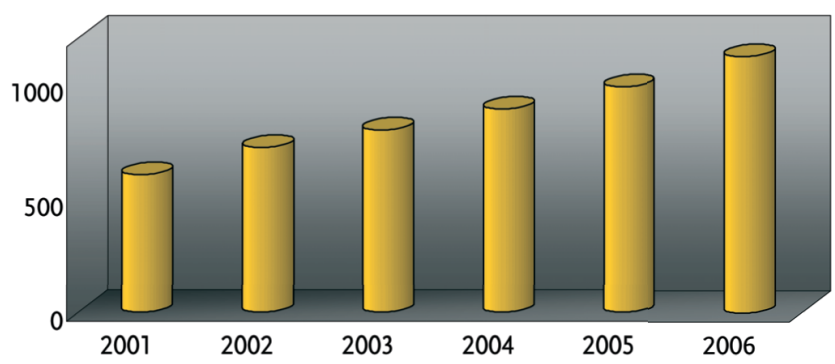

\section{EurJOC Facts}

- ISI Impact Factor (2006): 2.769 EurJOC ranks 18th of 56 journals included in the category "Chemistry, Organic" in the Journal Citation Reports ${ }^{\circledR}$ (ISI Web of Knowledge ${ }^{\mathrm{SM}}$ ). The Median Impact Factor in this category is 1.894 and the Aggregate Impact Factor is 2.564.

- Increased frequency in 2007: 36 Issues

- Owned and supported by the 13 national chemical societies of EUChemSoc (Editorial Union of Chemical Societies)

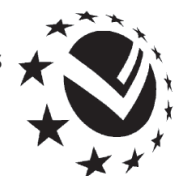

EUChemSoc

\section{EurJOC offers:}

- 1 RSS Feeds: be automatically informed of new articles as soon as they are published online in EarlyView

Citation tracking

- Backfiles (Liebigs Annalen 1832-1997)

- Attractive personal subscription rates for society members 